Culture et histoire dans l'espace roman

$6 \mid 2011$

Figures du pouvoir dans la littérature hispanoaméricaine

\title{
Splendeur et misère de Juan Manuel de Rosas, « el farmer » d'Andrés Rivera
}

\section{Marina Letourneur}

\section{(2) OpenEdition}

\section{Journals}

Édition électronique

URL : https://journals.openedition.org/cher/9922

DOI : $10.4000 /$ cher.9922

ISSN : 2803-5992

Éditeur

Presses universitaires de Strasbourg

Édition imprimée

Date de publication : 30 juin 2011

ISBN : 978-2-35410-033-9

ISSN : 1968-035X

Référence électronique

Marina Letourneur, "Splendeur et misère de Juan Manuel de Rosas, « el farmer » d'Andrés Rivera », reCHERches [En ligne], 6 | 2011, mis en ligne le 17 décembre 2021, consulté le 24 janvier 2022. URL http://journals.openedition.org/cher/9922 ; DOI : https://doi.org/10.4000/cher.9922

\section{cc) (1)(2)}

Ce(tte) œuvre est mise à disposition selon les termes de la Licence Creative Commons Attribution Pas d'Utilisation Commerciale - Partage dans les Mêmes Conditions 4.0 International. 


\title{
Splendeur et misère de Juan Manuel de Rosas, "el farmer » d'Andrés Rivera
}

\author{
MARINA LeTOURNEUR
}

Université d'Angers

$\mathrm{R}$ osas fut élu gouverneur de la Province de Buenos Aires en décembre 1829. Bénéficiant de «facultés extraordinaires», il devint « el Restaurador del orden y de las leyes» dans une Argentine fraîchement indépendante et marquée par l'instabilité politique et les guerres civiles. Après avoir refusé sa réélection en 1832 - celle-ci n'étant plus assortie des «facultés extraordinaires » dont il jouissait auparavant -, il revint à la tête de la province en 1834 et obtint, dans le dessein de «sauver la patrie», «la suma del poder político » qu'il exigeait pour une durée indéterminée. Dans le même temps, les provinces de la Confédération lui donnèrent le pouvoir de les représenter pour les relations extérieures. Il resta au pouvoir plus de vingt ans dans ce qui s'appelait alors la Confédération Argentine en dirigeant la Province de Buenos Aires d'une main de fer et en matant les différentes rébellions dans les autres Provinces jusqu'au 3 février 1852 (bataille de Caseros) où il perdit contre Urquiza, le caudillo de la Province d'Entre Ríos. Il dut alors s'exiler en Angleterre où il mourut en 1877.

La figure de Rosas apparaît dans En esta dulce tierra et La revolución es un sueño eterno, les deux romans de Rivera qui précèdent El farmer, puis dans Ese manco Paz (2003). Gregorio Cufré, le protagoniste de En esta dulce tierra, est traqué par la police politique de Rosas (la Mazorca) et doit se cacher chez une ancienne maîtresse rosiste en attendant la chute du tyran. Le roman évoque à plusieurs reprises le triste sort réservé aux opposants capturés par la Mazorca. Rosas est très brièvement mentionné à la fin de La revolución 
es un sueño eterno quand on apprend que "en 1839, la cabeza de Pedro Castelli, clavada en una pica por las triunfantes tropas del brigadier general Juan Manuel de Rosas, es ofrecida a la contemplación de los habitantes del poblado bonaerense de Dolores» (Rivera 2000:186).

Dans l'historiographie argentine, la figure de Rosas a longtemps été associée à la barbarie politique. Rosas fut considéré comme le premier despote de l'histoire du pays car il fut le premier à rester longtemps au pouvoir après avoir systématisé le recours à la violence et la traque sans relâche des opposants ${ }^{1}$. L'histoire argentine allait connaître d'autres régimes assimilables au rosisme, notamment la dictature militaire de 1976-1982 qu'évoque Rivera à travers l'histoire de Cufré dans En esta dulce tierra (1984).

Cependant, le courant révisionniste de l'historiographie argentine a réhabilité la figure de Rosas en mettant en avant son rôle de défenseur de la souveraineté nationale face aux deux grandes puissances de l'époque, la France et l'Angleterre ${ }^{2}$, et en soulignant son rôle d'organisateur de l'union nationale préalable à la mise en place de la Constitution. Le président Carlos Menem, qui possédait un portrait de Rosas dans sa geôle pendant la dernière dictature militaire, a fait rapatrier ses restes en 1989 lors d'une cérémonie solennelle, et Rosas figure sur les billets de 20 pesos depuis janvier 2000.

Dans El farmer, Rivera choisit d'aborder cette ancienne figure autoritaire dans la misère de l'exil, misère qu'il fait contraster avec la splendeur passée d'un personnage qui aspire à laisser son nom dans l'Histoire argentine.

\section{La misère de l'exil}

Dans ce court roman, Rivera fait de Rosas le narrateur principal et la focalisation est essentiellement interne. Un narrateur à la troisième personne - sorte de dédoublement du protagoniste - apparaît mais très rarement. Rivera donne ainsi à son roman un faux air de témoignage ou d'autobiographie. Il déclara à sa sortie en 1996 avoir choisi la première personne pour «ne pas juger», "pour essayer de comprendre» (Rodríguez 2004), et dit dans une entrevue:

1 L'un des premiers écrivains à avoir dépeint Rosas comme un tyran fut Sarmiento, son ennemi idéologique, dans la biographie de Facundo Quiroga rédigée pendant son exil au Chili.

2 En hommage à la bataille de Vuelta de Obligado (20/11/1845) où les troupes argentines ont triomphé des troupes anglo-françaises, le 20 novembre, "Día de la Sobranía Nacional», a été décrété férié en Argentine le 20/11/2010. 
No siento por él [Rosas] ninguna simpatía (como la tenía por Castelli) ni política, ni ideológica, ni humana. Pero tengo algunos puntos de contacto con Rosas. Cuando me lancé a escribir El farmer yo ya era un viejo, como Rosas. Rosas vivía en el exilio, yo también, en este país. Rosas estaba solo, y yo también. Entonces lo hice hablar, y lo hice hablar en primera persona, siguiendo una recomendación de Cesare Pavese. Nadie podía pensar que Rosas se iba a golpear el pecho hablando en primera persona, ni que yo iba a escribir un panfleto antirosista con Rosas hablando en primera persona (Lanese, Montero, Portela 2002).

De fait, Rivera choisit d'évoquer Rosas vers la fin de sa vie, alors qu'il a 78 ans et vit exilé en Angleterre. Dès l'incipit, il nous dépeint un Rosas qui mène une vie solitaire et austère, presque ascétique:

No fumo. No tomo vino ni licor alguno. Ni rapé. No asisto a comidas. No visito a nadie. No recibo a nadie. [...]

No voy al teatro. No paseo. Mi ropa es la de un hombre común.

En mis manos y en mi cara se lee, como en un libro abierto, cuál es mi trabajo durante los treinta santos días del mes.

Uso botas.

Mi comida es un pedazo de carne asada. Y mate.

No tengo mujer.

No ando de putas (Rivera 2002: 9).

Rosas, grand propriétaire terrien en Argentine, se définit comme un simple «campesino » dans son exil anglais : «dueño de una granja de apenas 37 hectáreas, de un rancho que sus vecinos no envidian ni codician» (14). Il dépend financièrement de quelques anciens amis et maintient une correspondance humiliante avec eux dans laquelle il se voit obligé de leur réclamer d'anciennes dettes dont ils ne sont jamais acquittés (57-58) ou de leur faire l'aumône (73). Il se sent trahi et abandonné de tous, même sa fille Manuelita, qui était presque son double - «Ella era Rosas» (75) -, s'est mariée contre son avis et ne lui rend plus visite. Il compare ce qu'il endure à la tragédie du Roi Lear de Shakespeare et, dans l'isolement de son exil, ressasse cet abandon dans un long soliloque: «Estoy solo, y hablo, para mí, en un frío mediodía británico./ Soy un hombre fuerte, y lloro, a veces, el olvido de los otros. ¿Por qué mi vejez no debe llorar, a veces, el olvido de los otros?» (61).

Il évoque à plusieurs reprises les circonstances qui ont entrainé son exil : la défaite contre les troupes d'Urquiza lors de la bataille de Caseros et le peuple dont il avait su se faire aimer qui lui a tourné le dos et crie Viva Urquiza après avoir crié Viva Rosas. Cette trahison l'affecte et il déclare: "Demoré 
una vida en reconocer la más simple y pura de las verdades patrióticas: quien gobierne podrá contar, siempre, con la cobardía incondicional de los argentinos» (28-29).

L'exil qu'il subit depuis vingt ans est une souffrance permanente: "¿Sabe alguien qué es el destierro? ¿Sabe alguien cuántos son veinte años de destierro?»(15-16). Il répond à son médecin anglais qui lui demande où il habite: "¿Le hablaron, a usted, de un lugar llamado destierro?» (70). Il se sent étranger dans son comté où il est un simple "farmer» anonyme. Son essence argentine ressort dans son quotidien, presque comme une revendication, car il se nourrit exclusivement de "carne asada " et de maté, et dans les allusions faites à la nourriture en général: «Aquí los ingleses toman mate de mi mate. Yo les descubro el zapallo y el dulce de leche. Y los saborean. [...] Los ingleses comen bistec. Yo asado» (55). Il semble reproduire à échelle réduite dans ce qu'il appelle son ranch ou sa ferme sa vie dans son estancia, et rêve, pendant sa sieste, qu'il parcourt les grandes étendues de la pampa sur son cheval.

Le roman se déroule sur une journée qui semble être une journée d'hiver type pendant laquelle il se passe peu de choses que le narrateur consigne toutefois brièvement: il nourrit ses animaux, se nourrit, boit, urine (une des descriptions les plus longues de sa vie quotidienne), fait la sieste, écrit. Il pense avec nostalgie à l'été argentin alors qu'il souffre du froid britannique. Pendant tout le roman, il réfléchit à sa situation d'exilé, se remémore le passé assis auprès du feu et pense à la mort qui le guette (11) et apparaît comme une menace permanente tout au long de ce récit divisé en différentes parties introduites par des sortes d'épigraphes. La plupart évoquent la mort et, tels des memento mori, rappellent à l'ancienne figure du pouvoir qu'il n'est pas éternel: "Cuídate de la noche/ Cuídate del día/ La vejez es inevitable/ La muerte, también». La mort semble s'approcher et un certain suspense s'installe. Quand bien même on découvre, à la fin du récit, que c'était un loup qui rôdait autour de la ferme, on sent le narrateur hanté par sa vieillesse inéluctable et par l'imminence de sa mort. Alors, comme Castelli dans La revolución es un sueño eterno, le Rosas de Rivera, confronté lui aussi à une situation d'échec et de disgrâce, ressasse le passé et dresse le bilan de sa vie.

\section{La splendeur passée}

Le narrateur fait sans cesse des allers-retours entre la monotonie du présent et le passé en évoquant souvent des épisodes glorieux qui renforcent la souffrance de son bannissement, alternant ainsi grandeur et décadence. 
Au début, le narrateur tarde à révéler son identité, et après s'être présenté comme un pauvre et vieux paysan du comté de Swanthling (sic) en GrandeBretagne, il déclare: "Y si pronuncio mi nombre por estos campos de la desgracia, ¿quién sabrá decir: ahí va un hombre cuyo poder fue más absoluto que el del autócrata ruso, y que el de cualquier gobernante en la tierra? / Soy Juan Manuel de Rosas» (10). Et le narrateur est persuadé que vingt ans après son départ, quelque part dans de quelconques tavernes en Argentine, des gauchos continuent à scander son nom ${ }^{(37)}$. Bien souvent, il évoque la figure qu'il représentait avec des expressions hyperboliques: «Yo que fui el guardián de los sueños de los otros» (12), «Yo soy la luz» (46), «Soy el Santo Padre» (24), «el Padrenuestro» (83).

Rivera décrit sommairement le Rosas de soixante-dix-huit ans; on sait juste qu'il se sent vieux et a besoin de sommeil, que son médecin lui dit de marcher pour faciliter sa circulation sanguine et irriguer son cerveau, qu'il lui arrive de pleurer, qu'il radote et n'a plus de femme. Dans d'autres romans où figure Rosas - En esta dulce tierra ou Ese manco Paz -, le personnage alors plus jeune et encore au pouvoir est toujours décrit avec un physique avantageux ${ }^{3}$. Dans El farmer, Rosas n'est jamais décrit physiquement du temps de sa splendeur, le narrateur autodiégétique se contente de faire contraster son état de vieillard avec la virilité et l'énergie sexuelle qui le caractérisaient et reflétaient sa puissance et son pouvoir: "Digo que los viejos lloran. [...] Y el llanto, en mí, es una larga y melancólica despedida a la energía de la edad viril» (31). La virilité du Rosas du passé transparaît notamment quand il évoque l'époque où il domptait les juments mais aussi dans ses rapports avec les femmes -qu'il s'agisse de son épouse, de sa maîtresse, ou d'anciennes concubines séduites par sa puissance (19). Rosas n'hésite pas à se comparer à un taureau et relate une anecdote où l'on voit qu'il mesurait sa supériorité envers certains de ses rivaux, ici Juan Lavalle, en comparant la taille de leur sexe (67).

Hormis Lavalle, deux autres figures rivales se détachent surtout dans ce récit : Urquiza son ennemi militaire et Sarmiento, son ennemi politique et idéologique. La situation d'exilé du Rosas de Rivera rappelle l'exil de Sarmiento au Chili alors que Rosas était au pouvoir. Les rôles s'inversent puisque le roman se passe en 1871 et qu'à ce moment-là Sarmiento est Président en Argentine depuis plus de trois ans. Le narrateur cite à plusieurs reprises Sarmiento, surtout lorsque ce dernier lui reconnaît un quelconque

3 «El hombre rubio, alto, sano, hermoso» (Rivera 2004: 19). 
mérite (12 et 90). Mais il cite aussi, dès le début du roman, ce commentaire de Sarmiento à son égard, extrait de Facundo: "Hace el mal sin pasión». S'ensuit alors une réflexion autour des notions de Bien et de Mal, le narrateur cherchant à démontrer que ce qui représentait le Mal pour Sarmiento, était un mal nécessaire selon lui: «El Mal, en mi boca y por mi brazo, fue orden y justicia» (13). La campagne du Désert - qui s'accompagna du massacre des Indiens qui ne se soumettaient pas à son autorité - permit, pour le narrateur, d'agrandir le territoire national et de pacifier la frontière avec lesdits Indiens. Quant à la traque puis la torture et l'assassinat des opposants hommes, femmes, et leurs enfants par la Mazorca, c'était selon lui le seul moyen d'assurer l'ordre et la justice. Rosas, "el Santo Padre», semble être le seul capable de distinguer réellement le Bien du Mal. Il dit que Sarmiento «Cree que hace El Bien» (26) et lui répond :

Hace el mal sin pasión, escribió de mí, el señor Sarmiento. Acepto eso. Y lo acepto porque soy argentino, y porque los argentinos, unitarios y federales, $y$ eso ya se dijo, somos puros cristianos.

Y el señor Sarmiento, que es argentino, escribió desde el silencio de un escritorio: Derrame sangre de gauchos, que es barata.

Que se escriba qué diferencia al general Rosas del señor Sarmiento (112-113).

Comme la plupart des personnages de Rivera, Rosas reste fidèle à ses principes. Le récit est d'ailleurs émaillé d'assertions qui étaient les consignes qu'il donnait à la population ${ }^{4}$. Ces consignes, reprises par le narrateur, semblent figées par l'écriture qui les réactualise. Rosas ne remet pas en question l'exercice de son pouvoir passé, il ne regrette en rien ses décisions. Il s'oppose toujours à Sarmiento qui voudrait "civiliser", c'est-à-dire européaniser un pays trop longtemps dominé par la barbarie des gauchos et des propriétaires terriens. Rosas donne son avis sur les révolutions européennes comme la Commune de Paris, ou sur les grèves des mineurs anglais. En cela le personnage est bien une création de Rivera, ancien syndicaliste et militant communiste, qui évoque même Marx sans le nommer (60). Le Rosas de Rivera se méfie des révolutionnaires depuis 1810. On peut lire dans le troisième chapitre qu'il se différencie des révolutionnaires de Maiqui auraient semé le doute et l'instabilité dans le pays: «Los jacobinos, con la Revolución de Mayo, nos empujaron al mundo de la enfermedad, de la disolución y de la duda. / Yo no me enfermo » (46).

4 «Lo que no se ve está fuera de la ley»(26), "El que está abajo respeta al que está arriba» (33), "Queda desautorizado lo que no autoricé» (53), "La vaca es vaca y no toro ( 65), «A los enemigos del orden, mazorca» (96), «La patria no es el hogar de la casualidad» (118). 
Le roman commence d'ailleurs par cette épigraphe: «Que en mi epitafio se lea: Aquí yace Juan Manuel de Rosas, un argentino que nunca dudó».

Cette épigraphe donne le ton du récit. Figure politique alors décriée, Rosas ne cherche pas à ce qu'on l'absolve de quoi que ce soit, il n'a aucun remord, et revendique même son importance historique. Et Rivera fait de lui un narrateur autodiégétique qui affirme son identité avec emphase puisque l'emploi du pronom personnel sujet "Yo» est presque systématique. En outre, à maintes reprises il répète «Soy Rosas», comme pour insister sur le fait qu'il est unique, voire exceptionnel, et qu'il continue à faire autorité: «Soy, aun solo, Juan Manuel de Rosas. / Invoco, nombrándome, lo que la patria me debe» (99). Le moment où il choisit de marquer son indépendance vis-à-vis de ses parents et décide de se faire appeler Rosas au lieu de Rozas est même évoqué, comme pour insister sur l'unicité de cet homme.

Rivera fait de Rosas un personnage complexe, à la fois englué dans une situation humiliante qui lui échappe mais aussi persuadé d'avoir toujours agi dans l'intérêt de son pays et d'être victime «d'une injustice, d'un crime, d'un péché de sang» (37). Le narrateur estime donc mériter de rester dans l'histoire argentine: « $\mathrm{Y}$, ahora, yo, gobernador-propietario de la provincia más extensa y rica de América, de la América española, estoy aquí en el condado de Swanthling, reino de la Gran Bretaña, [...] un desconocido para quienquiera que escuche, menos para la Historia. Y menos para mí» (13).

\section{«El nombre de la Historia.»}

"¿Soy el nombre de la Historia que se mira a ningún espejo, y habla con ningún espejo?» (25). Le traitement du temps dans ce récit interpelle car il est intimement lié à l'Histoire à laquelle veut appartenir Rosas. Bien que le référent soit historique, l'achronie ${ }^{5}$ caractérise ce récit et se manifeste de différentes façons. L'évocation des souvenirs de Rosas se fait souvent au passé, mais aussi au présent, temps de prédilection car il permet de refléter les états d'âme du narrateur, notamment sa souffrance d'exilé. Cet emploi du présent contribue à faire stagner le temps, ce que font aussi les nombreuses réitérations et reformulations qui rendent le présent interminable. C. Gilman dit à ce sujet:

Las formas textuales del padecimiento se revelan en las repeticiones, interrupciones, en los esfuerzos que entraña la posibilidad de narrar. [...]

5 Voir Genette qui montre comment le récit peut s'émanciper du temps de l'histoire et tendre alors vers l'achronie. 
Ese padecimiento deja sus marcas en una técnica narrativa que hace el presente interminable y que borra las posibilidades de una cronología fluida que se encamine hacia su desenlace. Se asiste a la eficacia controladora de un presente que parece durar perpetuamente (Gilman 1991: 60-61).

Rivera a par ailleurs souvent recours à l'ellipse car le narrateur fournit peu d'indicateurs temporels concrets comme les dates. Il choisit de ne préciser que celles qui lui importent ${ }^{6}$ en plus de celle du 27 décembre 1871 qui permet de fixer le temps diégétique du récit et de donner une perspective temporelle aux souvenirs du narrateur. D'autres faits historiques sont évoqués mais sans précision temporelle, le narrateur juxtapose différentes époques sans réelles transitions et les paragraphes sont seulement marqués par de longs espaces qui sont autant de pauses qui suspendent le récit. Ce flottement temporel, ces va-et-vient entre passé et présent rendent le temps élastique, relatif, et le narrateur Rosas se permet même un anachronisme quand il dit que selon Lord Palmerston, sa relation avec sa fille a inspiré Shakespeare quand il a écrit Le Roi Lear. Car Rosas - qui a connu la gloire par le passé et est à présent banni par ses concitoyens - pense qu'il restera malgré tout dans l'histoire de son pays: "yo, confinado aquí por la ingratitud de mis amigos y leal a la nobleza de mi origen y a mi casa, y al futuro que dirá, de mí, la palabra justa» (53). Il revient sur les critiques de Sarmiento à son égard et dit: «El señor Domingo Faustino Sarmiento [...] no le puso fecha a lo que escribió. / La verdad no vive en el calendario» (13). Il pressent qu'il marquera symboliquement le futur des Argentins: «Los argentinos darán mi nombre a su destino » (43). Marta Waldegaray souligne, concernant ces propos que: "No se trata propiamente del retorno de una idea, sino del anuncio de la persistencia de una marca política consistente en un modus operandi argentino que tendría en él su origen, según lo cree Rivera. Este vínculo de identidad cultural y política con los argentinos de todos los tiempos lo inmortaliza» (Waldegaray 2008: 3).

Le Rosas de Rivera réclame qu'on se penche sur lui et sur ce qu'il a fait pour l'Argentine: "tengo sobrado derecho a que se reflexione acerca de mí, de lo que fue y de lo que es Juan Manuel de Rosas» (61). Même si la mort rôde, il aspire métaphoriquement à une certaine éternité, comme on peut le lire dans l'épigraphe qui introduit le sixième chapitre et s'apparente à la fois

6 Les dates de ses élections ( $1^{\text {re }}$ élection comme gouverneur le 6 décembre 1829 et plébiscite des 26-27-28 Mars 1835), le 3 février 1852 (date de la défaite de Caseros et début de l'exil), le 31 octobre 1871 (messe en hommage aux Libres del Sur), le 23 octobre 1852 (mariage de Manuelita). 
à un onzième commandement divin et à une invocation : «Dios: Rosas no debe morir », ou lorsque le narrateur dit, à la fin du récit: "Yo soy como una novela de ese Shakespeare / Yo quedo» (119).

Toutefois, la crainte d'être oublié le taraude comme on le voit dans la dernière épigraphe qui ressemble à une supplique: "Patria, no te olvides de mí». Cette crainte met en lumière l'importance qu'accorde le narrateur à l'écriture tout au long du roman. Pour lui, quelle que soit la tournure que prennent les événements, les écrits restent. C'est pourquoi au moment d'embarquer pour l'exil il préféra prendre peu d'argent mais tint à emporter l'intégralité de ses archives, lesquelles lui rendraient justice devant l'Histoire: «Los papeles de mi archivo, que huelen a la más pestífera mierda que vientre alguno haya echado sobre la tierra, me absuelven y me honran ante el futuro» (34) .

Le narrateur lui-même se caractérise par une sorte de graphomanie. Quand il était au pouvoir il écrivait dix-huit heures par jour dans son bureau de Palermo. En exil, il continue à écrire: «Soy un campesino que escribe diez cartas diarias . Soy un campesino que escribe un Diccionario" (9). Écrire un Dictionnaire, c'est avoir une ambition totalisante vis-à-vis des mots, et le pouvoir politique de Rosas rendu caduque se mue pour l'exilé en un pouvoir d'écriture illimité.

Le roman, caractérisé par la transtextualité, est comme une mosaïque d'écrits constituée d'extraits de lettres privées et publiques dans lesquelles il donne son avis d'ancien dirigeant sur la situation politique, économique et sociale argentine ("Yo medito sobre la suerte de los argentinos sin mí », 61 ) et européenne. On trouve aussi des aphorismes, des citations plus ou moins apocryphes de Sarmiento et d'un anonyme "caballero francés», des allusions à Shakespeare. Les écrits lui permettent de continuer à exister publiquement puisqu'il est toujours question de lui: on parle de lui, on écrit sur lui, ou il donne son avis. La finalité est que le nom de Rosas, si souvent martelé, se fige dans ces écrits.

Ce qui caractérise le style et le contenu des écrits du narrateur ce sont les affirmations et sentences qui semblent irrévocables. Elles sont comme les stigmates d'un ancien pouvoir dictatorial et indiscutable. Les rares interrogations qui émaillent le récit renvoient à l'injustice et l'incompréhension que représente l'exil pour le narrateur, et au bien-fondé de la politique menée en Argentine par ceux qui lui ont succédé, notamment Sarmiento. 
Sa situation d'exilé lui rappelle évidemment celle de l'auteur de Facundo à qui il se compare: "Somos los dos mejores novelistas modernos de este tiempo " (23). Même si le général Rosas n’a pas les qualités littéraires de Sarmiento et n'arrive pas à publier, il s'autoproclame romancier, écrivain, et dit qu'en parlant de lui-même à de jeunes «doctorcitos", avec ses mots qu'il compare à des pépites d'or, il ne fait rien d'autre que leur révéler les secrets du roman moderne (24 et 25). Le narrateur semble reprendre à son compte des propos tenus par son auteur qui dit: «La literatura dice la verdad [...]. La historia la escriben los vencedores y la historia de los vencedores canoniza. La otra historia es tarea de la buena literatura.» (Speranza 1995: 186). Dans sa position de vaincu, le Rosas de Rivera règle ses comptes et tord le cou à ses détracteurs. Il révise l'histoire avant l'heure en choisissant d'écrire, comme romancier, sa propre histoire.

L'Histoire argentine occupe une place de choix dans l'œuvre de Rivera. L'auteur, qui refuse que certains de ses romans soient qualifiés d'historiques, fait le choix du narrateur autodiégétique et donc d'une focalisation interne ; en outre il privilégie l'histoire privée, parfois anecdotique, aux grands faits historiques auxquels les personnages qu'il met en scène sont associés. Ainsi, le «farmer» Rosas devient presque, dans son exil anglais, un héros tragique. Son expatriation de vingt ans, après autant d'années passées au pouvoir, lui font remettre en question les notions de Bien et de Mal - qu'il relativise, voire banalise - et qu'il pousse à leurs limites. Il bouleverse ainsi le discours de l'Histoire qui devient flou et fluctuant, à l'image de la figure de Rosas dans l'historiographie argentine, qui le considérera tantôt comme un défenseur des intérêts nationaux, tantôt comme le prototype de la barbarie et de la violence d'État.

\section{Bibliographie}

Genette, Gérard, 1972, Figure III, Paris, Seuil.

-, 1979, Fiction et diction, Paris, Seuil.

Gilman, Claudia, 1991, "Historia y poética del padecimiento en las novelas de Andrés Rivera", in Roland Spiller (ss dir.), La novela argentina de los años '80, Frankfurt am Main, Vervuet Verlag.

Lanese, Diego, Montero, Hugo, Portela Ignacio, 2002, Revista Sudestada ${ }^{\circ} 10$, "Andrés Rivera: "No puedo hacer otra cosa que escribir libros" ", julio de 2002: http://www.revistasudestada.com.ar/web06/article.php3?id_article=228 
Quattrocchi-Woisson, Diana, 1990, «Discours historique et identité nationale en Argentine», Vingtième siècle, vol. 28.

Rivera, Andrés, 1995, En esta dulce tierra, Buenos Aires, Alfaguara.

-, 2000, La revolución es un sueño eterno, Buenos Aires, Alfaguara, Punto de lectura.

-, 2002, El farmer, Buenos Aires, Alfaguara, Punto de lectura.

-, 2004, Ese manco Paz, Buenos Aires, Alfaguara, Punto de lectura.

Rodríguez, Julián, 2004, "Sobre El farmer y Andrés Rivera», Letras libres, junio de 2004 : http://www.letraslibres.com/index.php?art=9701

Sarmiento, Domingo Faustino, 1990, Facundo. Civilización y barbarie, Madrid, Cátedra (1re publication: 1845 ).

Speranza, Graciela, 1995, Primera persona (Conversaciones), Buenos Aires, Norma.

Waldegaray, Marta Inés, 2008, «Mito romántico y desencanto político en la figura de Juan Manuel de Rosas », in Petrich Perla, Premat Julio, Llombart María, Les sujets contemporains et leurs mythes en Espagne et en Amérique latine, Universitat d'Alacant, Université de Paris 8, Vincenne-Saint-Denis, p. 1-13. 